\title{
Transtek, InC.
}

\section{DOE/EE/15673-T.8}

5001 BAUM BLVD. - SUITE 799 - PITTSBURGH, PA 15213-1851 - PHONE: 1-412-802-8455 - FAX: 1-412-802-8456

\section{REGEIVED}

ОСТ 011998

Federal Grant DE-FG01-96EE15673

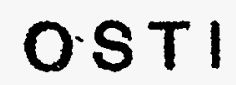

September 15, 1998

\section{FINAL TECHNICAL REPORT}

The objective of this project was to develop, design and build a system prototype to demonstrate the practicality of two-way, wireless through-the-earth communications between the interior of a mine and the surface. The system was to communicate data for process and environment monitoring and control, and provide real-time voice communication for emergency situations and for daily operations use.

The earth is a very poor medium for electromagnetic transmission. The difficulty in through-the-earth wireless information transmission is further compounded by restrictions imposed by intrinsic safety requirements in hazardous mine environments. The challenge was to communicate electromagnetically through the earth with a signal-to-noise ratio high enough to allow for message detection and comprehension.

We confirmed the commercial need for a wireless communications link between the interior of a mine and the surface in discussions with maintenance and management personnel of coal mines, as well as with mining equipment marketing organizations. The shortcomings of hardwire cable communications links have been studied extensively and are documented in published literature. Cables are vulnerable to deterioration and breakage in the hostile mine environment. The cost of cable installation is typically forty percent of the total system cost. The cable is unreliable and expensive to maintain. A reliable communication system will substantially increase the productivity of coal extraction, reducing coal price, and it will increase safety in mines and enhance rescue efforts to save lives of trapped miners in case of a mine disaster.

In a preliminary step, we visited mines in Pennsylvania and Ohio and we made electric noise measurements. We developed a mathematical model for electromagnetic communication through the earth. The model includes critical parameters such as the electric conductivity of the earth, the depth of the mine, the eccentricity between the transmitting and receiving antennas axes, the antennas dimensions and the transmitting voltage and current magnitudes and frequency. The model predicts the received magnetic field intensity and the induced voltage magnitude in the receiving antenna as well as the stored energy in the transmitting antenna which is a critical factor in intrinsic safety determination. We conducted experiments in an actual mine to evaluate the validity of the model and we confirmed its correctness. Combining this model with available data of electric noise in mine environments, we determined that through-the-earth wireless communication was feasible provided we employed sophisticated signal-to-noise enhancement techniques.

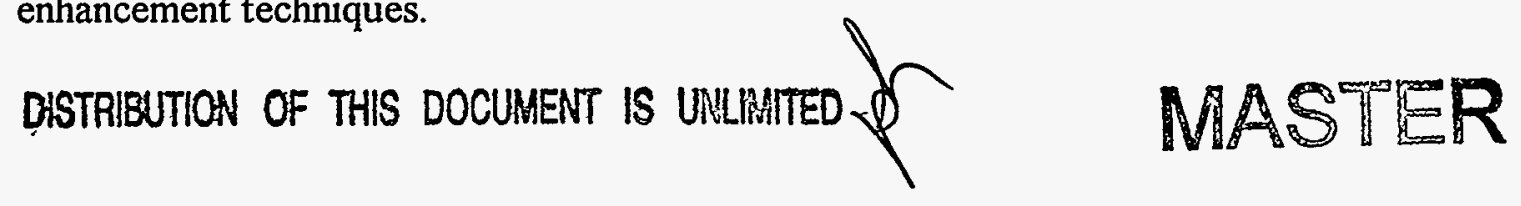




\section{DISCLAIMER}

This report was prepared as an account of work sponsored by an agency of the United States Government. Neither the United States Government nor any agency thereof, nor any of their employees, makes any warranty, express or implied, or assumes any legal liability or responsibility for the accuracy, completeness, or usefulness of any information, apparatus, product, or process disclosed, or represents that its use would not infringe privately owned rights. Reference herein to any specific commercial product, process, or service by trade name, trademark, manufacturer, or otherwise does not necessarily constitute or imply its endorsement, recommendation, or favoring by the United States Government or any agency thereof. The views and opinions of authors expressed herein do not necessarily state or reflect those of the United States Government or any agency thereof. 


\section{DISCLAIMER}

Portions of this document may be illegible in electronic image products. Images are produced from the best available original document. 
We designed and built transmitters and receivers - combined into transceivers - to evaluate the practicality of such a communications system. This included the design of proprietary signal processing modules, power amplifiers, and antennas. The system contains hardware and software. The software was entered into microprocessors and . DSPs (Digital Signal Processors) converting it into firmware. Consequently, the equipment is self contained and of practical dimensions.

During the design and development period, we tested portions of the system under simulated conditions and made modifications and adjustments as was necessary. We then tested the finished prototype in an actual mine in Western Pennsylvania. The test results were entirely satisfactory. The clarity of the real-time voice communication was extremely high. To test the data communication aspect, we connected to our transceiver inside the mine a temperature sensor used in coal mines. Upon periodic requests initiated at the transceiver on the surface of the mine, we received readings of the temperature inside the mine displayed on a monitor on the surface of the mine.

We had envisioned that in large mines (mines that are several miles long and several miles wide) data and voice communication would be carried inside the mine through hardwired networks to our transceiver inside the mine from where it would be transmitted, using our system, to the surface. During our visits to the mines, we found that for complete mine coverage, large areas inside the mine must communicate through wireless means. It is far too expensive and in some cases physically impractical to install wires to all locations where communications is needed. Wireless systems for this purpose that are available on the market are not satisfactory to mine operators.

Therefore, we developed (supported, in part, with funds granted by the Commonwealth of Pennsylvania) a wireless in-mine communications system. This system requires a certain amount of wire installation, but considerably less than is required by competing systems, and uses wireless two-way radios for communication through our network between remote locations. We have received an order for this system from a mine in Pennsylvania. The decision makers for this acquisition evaluated our system together with competing systems and found ours superior in wireless area coverage per quantity of installed wire and in clarity of voice. And, using sound business practices, our system was competitively priced.

When our through-the-earth communications system is interfaced with our inmine communications system, miners underground will be able to communicate with one another as well as with the surface.

In building our equipment, we employed rugged, professional fabrication technologies including printed circuits and reliable harnesses, mountings, and interconnections. We used waterproof connectors and manufactured rugged, waterproof enclosures. This is necessary to protect the circuitries from the high humidity inside mines. Our systems are adequate for hard rock mines such as the lime stone mine that purchased our in-mine communications system. But, in their present states, the systems 
do not meet MSHA (Mine Safety and Health Administration) specifications for hazardous coal mines.

Throughout the period of this project, we worked closely with the Company that will market our products in the US and abroad. This Company is a major player in the coal mining industry. It is a highly respected distributor and installer of mining equipment. The Company has offices in countries on all continents and is well positioned in the worldwide market.

In summary, we successfully demonstrated the feasibility of our concept and the marketability of our product. Additional work must be done to make the product suitable for, and marketable to, the coal mining industry. The product must be designed to be intrinsically safe and explosion-proof. Based on our present system design and performance, this is possible to accomplish. The need for our product is well documented. The marketing network is in place through our strategic alliance with a worldwide marketer. 